\title{
Mixed Valency versus Covalency in Rare-Earth Core-Electron Spectroscopy
}

\author{
G. Kaindl \\ Institut für Atom- und Festkörperphysik, Freie Universität Berlin, D-1000 Berlin 33, Germany \\ G. K. Wertheim \\ AT\&T Bell Laboratories, Murray Hill, New Jersey 07974 \\ and \\ G. Schmiester and E. V. Sampathkumaran ${ }^{(a)}$ \\ Institut für Atom- und Festkörperphysik, Freie Universität Berlin, D-1000 Berlin 33, Germany
}

(Received 18 November 1985; revised manuscript received 26 September 1986)

\begin{abstract}
A comparison of photoemission from shallow core levels with deep core-level spectroscopies allows a distinction between mixed valency and covalency in rare-earth systems. The determinative parameter distinguishing these spectroscopies is the Coulomb interaction between the core hole and the $4 f$ shell. This approach confirms that the compound $\mathrm{CeF}_{4}$ is tetravalent, but does not deny the covalent admixture of $4 f$ character into the valence band.
\end{abstract}

PACS numbers: $71.50 .+\mathrm{t}, 75.20 . \mathrm{Hr}, 79.60 .-\mathrm{i}$

Recent efforts to understand the properties of $\mathrm{Ce}$ and its compounds have tended to blur the concept of mixed valency ${ }^{1-3}$ originally used to describe compounds of the heavier rare earths, which exhibit interconfiguration fluctuations and, in effect, have a narrow $4 f$ level as well as a broad $5 d$ band at the Fermi level. This model of the electronic structure is not directly applicable to $\mathrm{Ce}$, in which the $4 f$ states have much greater radial extent and may participate in bonding. Core-electron photoemission and $L$-edge x-ray-absorption (XA) spectra of many Ce compounds, including insulators, were found ${ }^{4-6}$ to be similar to those of acknowledged mixed-valence compounds, leading to the suggestion that there is no fundamental distinction between these materials. The theory of Gunnarsson and Schönhammer ${ }^{7}$ (GS) showed that such spectra arise whenever a $4 f$ level is coupled to a conduction band in the initial state. Later it was shown ${ }^{8,9}$ that even covalent mixing of $4 f$ character into a filled band ${ }^{10}$ is sufficient to produce core-electron spectra resembling those of mixed-valence compounds. Such covalent materials are, however, conceptually distinct from the mixed-valence compounds mentioned above. It seems, therefore, that, although core-electron photoemission and $L$-edge spectra provide a new tool for the measurement of band mixing, they cannot distinguish between covalency and mixed valency. However, it does not seem necessary to abandon this useful distinction simply because of the inability to realize it in one type of measurement.

In this work we use core and valence $\mathrm{X}$-ray photoelectron spectroscopy (XPS), as well as $L$-edge XA spectra, of the transparent, ionic compound $\mathrm{CeF}_{4}$ to provide a new criterion for the identification of mixed-valence behavior in photoelectron spectroscopy. The $\mathrm{CeF}_{4}$ was prepared by the reaction of $\mathrm{CeF}_{3}$ with purified $\mathrm{F}_{2}$ gas at $300^{\circ} \mathrm{C}$ and 200 atm for three days in a closed $\mathrm{Ni}$ container; it resulted in pure $\mathrm{CeF}_{4}$ with the $\mathrm{CrF}_{4}$ monoclinic structure. ${ }^{11}$ Magnetic susceptibility measurements set an upper limit of $0.1 \%$ for $\mathrm{CeF}_{3}$ impurities. $L_{\mathrm{III}}$-edge XA spectra were taken at the EXAFS-II beam line at HASYLAB (Hamburger Synchrotronslabor) in Hamburg, by use of a $\mathrm{Si}(111)$ double-crystal monochromator with a rocking-curve width of $1.4 \mathrm{eV}$ (FWHM) at 6-keV photon energy. In the XA measurements, external pressures up to $\simeq 300 \mathrm{kbar}$ could be applied to the sample by use of an opposed-anvil device with diamond anvils in combination with a $\mathrm{Be}$ gasket. Homogeneous absorbers were prepared from $\mathrm{CeF}_{4}$, which was finely powdered in Ar atmosphere and mixed with epoxy. The XPS data were obtained with a Hewlett-Packard model HP-5950A spectrometer with a base pressure of $10^{-10}$ Torr, employing monochromatized $\mathrm{Al} K \alpha$ radiation, with a total system resolution of $0.55 \mathrm{eV}$. The surface of $\mathrm{CeF}_{4}$ was prepared in situ by abrasion with a diamond file. Surface contamination was periodically checked by our recording the $1 s$ spectra of oxygen and carbon.

The important new information is contained in the shallow core-level photoemission spectra, which appear in the valence-band regions of $\mathrm{CeF}_{3}$ and $\mathrm{CeF}_{4}$ in Fig. 1. The spectra are shown with the $\mathrm{F} 2 p$ valence bands aligned. The zero of binding energy corresponds approximately to the edge of the empty conduction band in $\mathrm{CeF}_{3}$. The signal due to the localized $\mathrm{Ce} 4 f$ level is readily identified just above the valence band in $\mathrm{CeF}_{3}$. In $\mathrm{CeF}_{4}$ the $4 f$ states are empty, and the other core levels are shifted to greater binding energy, in accord with the increased valency. The weak $4 f$ signal that can be discerned above the valence band is associated with the surface layer in which the $\mathrm{Ce}$ atoms do not have a full complement of $\mathrm{F}$ neighbors. The surface nature of this 


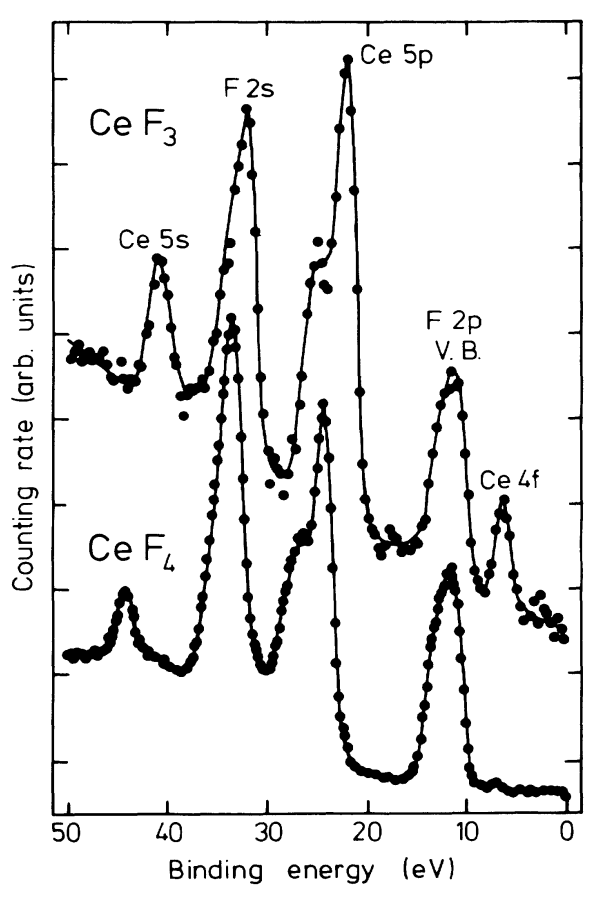

FIG. 1. XPS spectra of the valence-band regions of $\mathrm{CeF}_{3}$ and $\mathrm{CeF}_{4}$. The lines are drawn as a guide to the eye.

signal was confirmed by spectra at lower photon energy, where the escape depth is significantly smaller and the $4 f$ signal correspondingly greater. The most interesting feature is the Ce $5 s$ level in $\mathrm{CeF}_{4}$. In consists of a single component without a significant contriburion at the energy characteristic of $\mathrm{Ce}^{3+}$, other than expected on the basis of the weak $4 f$ signal from the surface; it has a narrow width because there is no $5 s-4 f$ multiplet interaction. The absence of a significant $\mathrm{Ce} 5 s$ signal at the $\mathrm{Ce}^{3+}$ position proves that the compound contains only $\mathrm{Ce}^{4+}$ ions, and can behave like an ionic, integer-valence system even in a fast, high-energy spectroscopy.

The picture changes dramatically when deeper core levels are photoionized. The data (see Fig. 2) show three spin-orbit-split doublets with distinct shapes, labeled $A_{1}$, $B_{1}$, and $C_{1}$, corresponding to different final-state configurations; they are populated by configuration interaction in the presence of a core hole. ${ }^{7}$ The spectrum is qualitatively similar to that of $\mathrm{CeO}_{2},{ }^{6,12}$ but the $A_{1}-C_{1}$ separation is $13.5 \mathrm{eV}$ as against $15.8 \mathrm{eV}$ in $\mathrm{CeO}_{2}$, and the $A_{1}$ $B_{1}$ separation is also much smaller. The assignment of these spectral components to specific $4 f$ configurations has been discussed extensively and quite controversially for $\mathrm{CeO}_{2} \cdot{ }^{6,12-14}$ There is general agreement that the high-energy doublet $\left(A_{1}\right)$ corresponds to a final state with predominant $4 f^{0}$ configuration: It lies at the largest binding energy and is narrow, indicating that there is no $3 d-3 f$ multiplet interaction. Since the $5 d$ band is empty, the core hole is screened only by the polarization

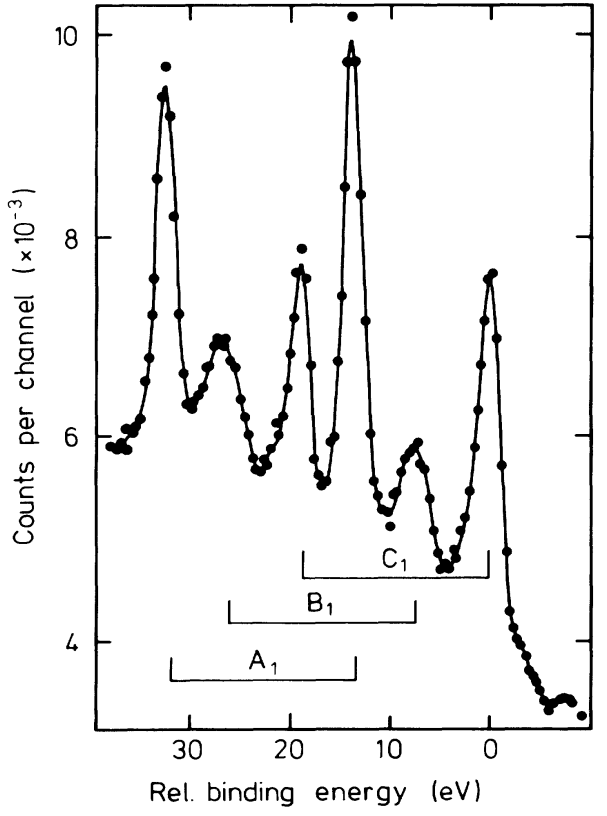

FIG. 2. Ce $3 d$ XPS spectrum of $\mathrm{CeF}_{4}$; the three spinorbit-split doublets are shown as bar diagrams. The lines are drawn as a guide to the eye.

of the neighboring fluorine ions. The $B_{1}$ and $C_{1}$ doublets, which lie at smaller binding energies, are considered as signatures of final states with strong mixing of $4 f^{1}$ and $4 f^{2}$ configurations, which are populated under the influence of the core-hole potential in the final state and the $4 f$ hybridization in the initial state. ${ }^{6,9,13-15}$ The spectra have been theoretically described by use of a modification of the GS model, ${ }^{7}$ which was extended in Refs. 6 and 9 to insulators. Physically speaking, the $B_{1}$ and $C_{1}$ doublets are produced by charge transfer from the F $2 p$ valence band to the empty $4 f$ level, made possible by the admixture of $4 f$ character into the $\mathrm{F} 2 p$ valence band in the initial state. The fact that the $B_{1}$ and $C_{1}$ doublets have relatively greater intensity in $\mathrm{CeO}_{2}$ follows simply from the greater covalency of that compound. We will not address the problem of the determination of the weights of the $4 f^{0}, 4 f^{1}$, and $4 f^{2}$ components of the final states $B_{1}$ and $C_{1}$ in the present paper. The essential point is that the spectrum in Fig. 2 shows a great deal of occupied localized $4 f$ character in the final state, which is in contrast to the findings from the shallow $5 s$ and $5 p$ core levels (Fig. 1).

It is apparent that the strong signals from final states with $4 f^{1}$ and/or $4 f^{2}$ configurations appear only when a deep, but not when a shallow, core level is excited, even though both excitations are "fast," or equivalently high energy as compared to typical covalent mixing energies. The relevant difference between $5 s$ and $3 d$ photoionization is found in the effect of the core hole on the $4 f$ shell. The $5 s$ charge lies largely outside the $4 f$ wave function, 
while the $3 d$ orbital is situated almost completely within it. According to the equivalent-cores approximation, in the final state with a $3 d$ hole, the $4 f$ level has the binding energy appropriate for the $Z+1$ ion, $\mathrm{Pr}^{4+}$, while $5 s$ ionization largely preserves the radial character and $4 f$ binding energy of the initial-state ion. The perturbation produced by a $5 s$ (or $5 p$ ) hole, while small, is not entirely negligible. It becomes important, e.g., when the $4 f$ system is of mixed valence in the initial state. In this case the $5 p$ photoemission, e.g., from mixed-valence TmSe, clearly shows contributions from both $4 f^{12}$ and $4 f^{13}$ final states. ${ }^{16}$ Photoemission from the $5 s$ and $5 p$ shells therefore provides a sensitive means of distinguishing the marginally stable $4 f$ configurations, which typify the classical mixed-valence systems, ${ }^{1}$ from systems with covalent admixtures into filled bands.

We have also studied the XA spectra at the $L_{\text {III }}$ threshold of $\mathrm{CeF}_{4}$ at external pressures up to $267 \mathrm{kbar}$ and for comparison of $\mathrm{CeF}_{3}$. The data are presented in Fig. 3. $L$-edge XA spectra of metallic rare-earth compounds are closely related to deep-core-electron photoemission spectra, because the final states have similar screening configurations, i.e., the partially filled $5 d$ band provides metallic screening in XPS, similar to that provided by the electron excited in the edge absorption process. This simplification does not apply to insulators, in which final-state screening in XPS is provided by polar-

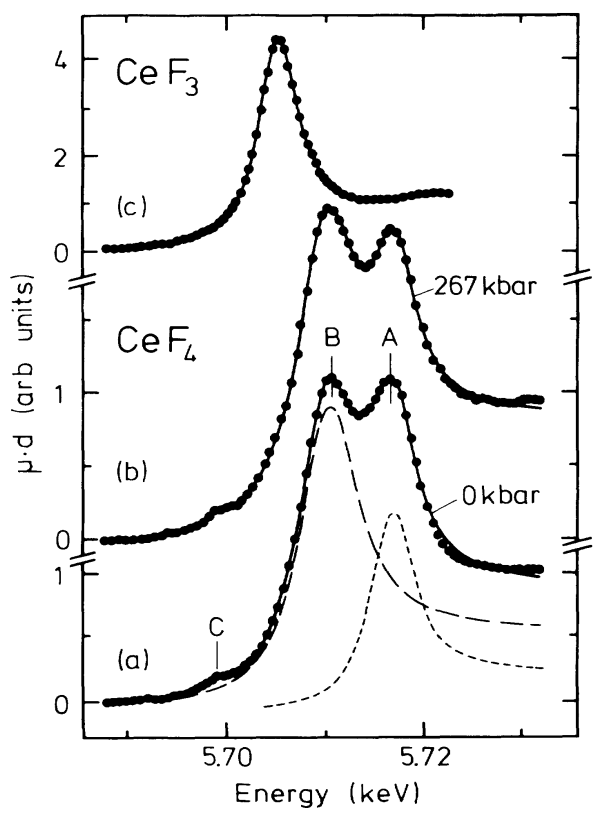

FIG. 3. Ce $L_{\text {III }} \mathrm{XA}$ spectra of $\mathrm{CeF}_{4}$ (a) at ambient pressure, (b) at $267 \mathrm{kbar}$, and (c) of $\mathrm{CeF}_{3}$. The solid lines through the data points are the results of least-squares fits. The two intense components $(\mathrm{A}, \mathrm{B})$ are given by dashed lines in the $\mathrm{CeF}_{4}$ spectrum. ization of the neighboring anions. The final states prepared by the two techniques in insulators consequently differ in a more profound way than in metallic systems.

In $\mathrm{CeF}_{3}$ [Fig. 3(c)] we observe a single "white line" representing excitation into the empty $5 d$ band, producing a fully screened $4 f^{1} 5 d$ state. The empty $4 f^{2}$ state lies above the top of the valence band. The data are well fitted by the combination of a Lorentzian to represent the white line and an arctangent function for the excitation into extended $d$ states, both convolved with a Gaussian resolution function. The $\mathrm{CeF}_{4}$ spectrum [Fig. 3(a)] contains two major lines (A and B) as well as a very weak feature (C) at smaller excitation energy. It is in most respects similar to those of $\mathrm{CeO}_{2}{ }^{17}$ and $\mathrm{Ce}\left(\mathrm{SO}_{4}\right)_{2}{ }^{18}$; the weak fourth component between lines $\mathrm{B}$ and C, reported previously in the $L_{\mathrm{III}}$ spectrum of $\mathrm{CeO}_{2}$, has also been observed in some of the $\mathrm{CeF}_{4}$ absorbers studied here. It is absent in the spectrum presented in Fig. 3; there is thus strong indication that this line, when observed, is due to $\mathrm{Ce}^{3+}$ impurities. A fit with a set of edge spectra of the type described above leads to the three components (A, B, C) shown by dashed lines in Fig. 3. The one at largest excitation energy is narrow and corresponds to a state with predominantly $4 f^{0} 5 d$ configuration produced by the dipole excitation process. The other two peaks (B and C) at smaller excitation energies are again produced by charge transfer from the ligand $2 p$ band to the $4 f$ level corresponding to final states that are strong mixtures of $4 f^{1}$ and $4 f^{2}$ configurations. ${ }^{15}$ Spectral feature $\mathrm{A}$ is relatively more intense in $\mathrm{CeF}_{4}$ at ambient pressure as compared to $\mathrm{CeO}_{2}$, which is considered a consequence of the higher covalency of the latter compound. The relative intensity of component $A$ decreases in $\mathrm{CeF}_{4}$ as a function of external pressure in an approximately linear way, the decrease in intensity amounting to $=7 \%$ at $267 \mathrm{kbar}$ [see Fig. 3(b)]. This observation is in full agreement with the given assignment, since covalency is expected to increase with increasing pressure.

We conclude from shallow core-level XPS that $\mathrm{CeF}_{4}$ is a tetravalent compound, a result in agreement with our susceptibility and recent soft- $\mathrm{x}$-ray absorption measurements. ${ }^{19}$ We find further that deep-core-level spectroscopies of $\mathrm{CeF}_{4}$ and similar compounds $\left[\mathrm{CeO}_{2}\right.$, $\left.\mathrm{Ce}\left(\mathrm{SO}_{4}\right)_{2}\right]$, are dominated by charge transfer into strongly perturbed, localized final-state $4 f$ levels. Charge transfer is mediated by $f$ character hybridized into delocalized valence bands, and results in spectra with components corresponding to a number of different $4 f$ occupancies in the final state. These spectra resemble closely those of mixed-valence systems with interconfiguration fluctuations. In contrast, excitation of electrons from shallow core levels, e.g., $5 s$ and $5 p$, provides only a weak perturbation, which is not sufficient to cause considerable charge transfer from ligand orbitals to a local- 
ized $4 f$ level in the final state. However, in mixedvalence systems with a fractionally occupied $4 f$ level in the initial state, the perturbation is sufficient to produce two final states separated by the core-hole- $4 f$ Coulomb energy. This provides the opportunity to distinguish between covalent and mixed-valence materials, and still maintain the original significance of the term "mixed valency."

One of the authors (G.K.W.) is indebted to the Alexander von Humboldt foundation for an award under whose auspices this work was broutht to completion. We wish to thank G. Wortmann for his assistance in the initial $L$-edge measurements and D. Lentz for his advice in sample preparation. The work at the Freie Universität Berlin was supported by the Bundesminister für Forschung und Technologie, under Project No. 05-313AXB/B3-17.

\footnotetext{
(a) Permanent address: Tata Institue of Fundamental Research, Bombay 400005, Maharashtra, India.

${ }^{1}$ C. M. Varma, Rev. Mod. Phys. 48, 219 (1976).

${ }^{2} \mathrm{P}$. Wachter, in Valence Instabilities, edited by P. Wachter and H. Boppart (North-Holland, Amsterdam, 1982), p. 145.

${ }^{3}$ See Proceedings of the International Conference on Valence Fluctuations, Cologne, 1984, J. Magn. Magn. Mater. 47-48 (1985).

${ }^{4}$ R. D. Parks et al., Phys. Rev. B 28, 3556 (1983).
}

5J. Röhler, D. Wohlleben, J. P. Kappler, and G. Krill, Phys. Lett. 103A, 220 (1984), and references therein.

${ }^{6}$ E. Wuilloud, B. Delley, W.-D. Schneider, and Y. Baer, Phys. Rev. Lett. 53, 202 (1984); A. Fujimori, Phys. Rev. Lett. 53, 2518 (1984).

${ }^{7}$ O. Gunnarsson and K. Schönhammer, Phys. Rev. B 28 , 4315 (1983).

${ }^{8}$ B. Delley and H. Beck, J. Phys. C 17, 4971 (1984).

${ }^{9}$ W.-D. Schneider, B. Delley, E. Wuilloud, J.-M. Imer, and Y. Baer, Phys. Rev. B 32, 6819 (1985).

${ }^{10}$ D. D. Koelling, A. M. Boring, and J. H. Wood, Solid State Commun. 47, 227 (1983).

${ }^{11} \mathrm{Yu}$. M. Kiseleu, L. I. Martyneko, and V. I. Spitsyn, J. Inorg. Chem. (USSR) 20, 998 (1975)

${ }^{12}$ A. F. Orchard and G. Thornton, J. Electron Spectrosc. Relat. Phenom. 10, 1 (1977).

${ }^{13}$ A. Fujimori, Phys. Rev. B 28, 2281, 4489 (1983).

${ }^{14}$ A. Kotani, H. Mizuta, T. Jo, and J. C. Parlebas, Solid State Commun. 53, 805 (1985).

${ }^{15}$ T. Jo and A. Kotani, Solid State Commun. 54, 451 (1985), and J. Magn. Magn. Mater 52, 396 (1985).

${ }^{16} \mathrm{M}$. Campagna, E. Bucher, G. K. Wertheim, D. N. E. Buchanan, and L. D. Longinotti, Phys. Rev. Lett. 32, 885 (1974).

${ }^{17} \mathrm{~K}$. R. Bauchspiess et al., in Valence Fluctuations in Solids, edited by L. M. Falicov, W. Hanke, and M. B. Maple (NorthHolland, Amsterdam, 1981), p. 417.

${ }^{18}$ A. Bianconi, A. Marcelli, M. Tomellini, and I. Davoli, J. Magn. Magn. Mater 47\& 48, 209 (1985).

${ }^{19}$ G. Kalkowski et al., Phys. Rev. B 32, 2717 (1985). 